\title{
Disfiguring Annular Sarcoidosis Improved by Adalimumab
}

\author{
C.A. Kaiser A. Cozzio G.F.L. Hofbauer J. Kamarashev \\ L.E. French A.A. Navarini
}

Department of Dermatology, University Hospital of Zurich, Zurich, Switzerland

\section{Key Words}

Cutaneous sarcoidosis - Systemic sarcoidosis - Sarcoidosis treatment - Adalimumab . TNF- $\alpha$ antagonist

\begin{abstract}
Depending on the location, dermatoses can produce blemishes that severely impair quality of life and require highly effective treatment that is otherwise used for extensive skin involvement. We report the case of a 39-year-old, otherwise healthy male disfigured by an $8 \times 7-\mathrm{cm}$ hypopigmented and centrally atrophic annular plaque with erythematous indurated borders in an area of scar tissue on his forehead. Skin biopsies revealed noncaseating granulomas, and hilar involvement was identified, leading to the diagnosis of systemic sarcoidosis stage II with cutaneous involvement. The lesions proved resistant to multiple therapies, but responded within 4 months to adalimumab with regression of the lesion and inflammatory infiltrate. The visual analogue scale of disease activity decreased from 7/10 to 3.5/10, and the Dermatology Life Quality Index from 16/30 to 3/30 points. In conclusion, TNF-a inhibition can control inflammation and disfigurement by cutaneous sarcoidosis and restore quality of life.
\end{abstract}

\section{Case Report}

A 39-year-old man developed an $8 \times 7$-cm hypopigmented and centrally atrophic plaque with erythematous indurated borders in an area of scar tissue on his forehead (fig. 1a). On the occipital scalp he presented an erythematous brownish plaque resulting in cicatricial alopecia, as well as multiple brownish patches and papules on the trunk and shins. Skin biopsies from forehead, scalp and the pretibial area showed inflammatory non-caseating granulomas (fig. 1b). Chest X-ray revealed bilateral hilar adenopathy, nodules and reticular opacities, while pulmonary function tests indicated an obstructive ventilation disorder with physiologic diffusion capacity for carbon monoxide. A diagnosis of systemic sarcoidosis stage II with cutaneous and lung involvement was made.

Prior to presentation, topical tacrolimus, systemic prednisolone, chloroquine, isotretinoin and acitretin had been unsuccessful. At our department, $20 \mathrm{mg}$ methotrexate weekly s.c. initially combined with systemic steroids did not improve the sarcoid plaques over 5 months. Subsequently, adalimumab was started at $80 \mathrm{mg}$ as a first dose and then at $40 \mathrm{mg}$ every second week. Methotrexate was stopped 
after 1 month of overlapping therapy due to elevated transaminases. Within 4 months the disfiguring forehead plaque regressed and flattened out, enabling camouflage of the remnant skin changes (fig. 1c). The subjective disease activity as measured by the visual analogue scale score decreased from $7 / 10$ to 3.5/10 points within 4 months, and the Dermatology Life Quality Index (DLQI) dropped from 16/30 to 3/30. A follow-up biopsy of the border of the forehead lesion revealed almost complete clearance of lymphocytic infiltrates and complete absence of granulomas.

\section{Discussion}

TNF antagonists have proven useful and safe for a number of cutaneous diseases such as psoriasis and pyoderma gangrenosum. For sarcoidosis, TNF- $\alpha$ targeting has been found to be effective in isolated cases [1]. Because inflammatory granulomas depend on TNF- $\alpha$ signaling [2], there is a rationale for its blockade. Even before biologics became available, thalidomide - a compound with anti-TNF- $\alpha$ activity - had been used successfully [3]. The skin involvement in our case was below $2 \%$ of body surface - an extent that would not qualify for biologic therapy, had he, for example, suffered from psoriasis. However, due to the intense impairment of quality of life by the disfiguring plaques as measured by DLQI, as well as because of pulmonary sarcoidal affection, the decision to use a biologic was deemed reasonable and reimbursable by the patient's health insurance. Whether this strategy should be adopted for all patients with sarcoidosis and would be affordable is up to debate.

In regard to side effects, TNF-blocking agents may be superior to most treatments described for sarcoidosis such as steroids, methotrexate, thalidomide, antimalarials, azathioprine, cyclosporin A, leflunomide and cyclophosphamide. Interestingly, as described for psoriasis [4], TNF antagonists have been reported to not only treat, but also induce cutaneous or systemic sarcoidosis [5]. Taken together, in selected cases, TNF- $\alpha$ inhibition may be beneficial for the treatment of cutaneous sarcoidosis with or without systemic involvement.

\section{Disclosure Statement}

Funding/support: This study was supported by the Department of Dermatology, University Hospital of Zürich. Financial disclosure: None of the authors have any relevant financial relationships to this work. 

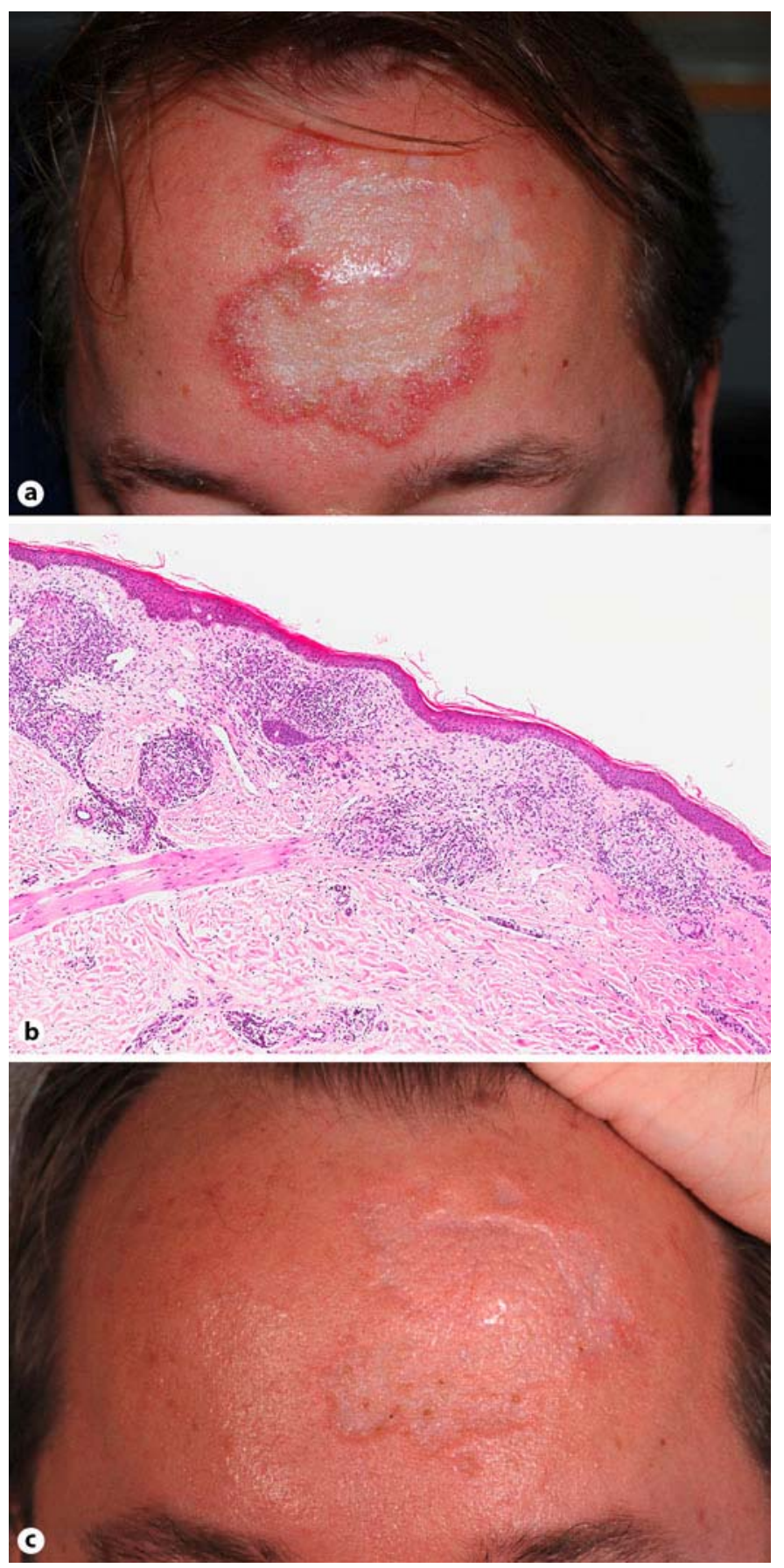

Fig. 1. a Hypopigmented and atrophic lesion with indurated erythematous border at diagnosis. b Histopathology before treatment with adalimumab (original magnification 20x). c Lesion after 4 months of treatment with adalimumab. 


\section{References}

-1 Heffernan MP, Anadkat MJ: Recalcitrant cutaneous sarcoidosis responding to infliximab. Arch Dermatol 2005;141:910-911.

2 Amiri P, Locksley RM, Parslow TG, et al: Tumour necrosis factor alpha restores granulomas and induces parasite egg-laying in schistosome-infected SCID mice. Nature 1992;356:604-607.

-3 Antoniu SA: Targeting the TNF-alpha pathway in sarcoidosis. Expert Opin Ther Targets 2010;14:21-29.

$\checkmark 4$ Cohen JD, Bournerias I, Buffard V, et al: Psoriasis induced by tumor necrosis factor-alpha antagonist therapy: a case series. J Rheumatol 2007;34:380-385.

5 Gifre L, Ruiz-Esquide V, Xaubet A, Gomez-Puerta JA, Hernandez MV, Sanmarti R: Lung sarcoidosis induced by TNF antagonists in rheumatoid arthritis: a case presentation and a literature review (in Spanish). Arch Bronconeumol 2011;47:208-212. 